\title{
MiR-410 Down-Regulates the Expression of Interleukin-10 by Targeting STAT3 in the Pathogenesis of Systemic Lupus Erythematosus
}

\author{
Dongmei Liu Na Zhang Xiaomei Zhang Muting Qin Youdan Dong Lei Jin
}

Department of Rheumatology and Immunology, Shengjing Hospital of China Medical University, Shenyang, People's Republic of China

\section{Key Words}

Systemic lupus erythematosus (SLE) • MiR-410 • Interleukin-10 (IL-10) • STAT3 • CD3+ T cells

\begin{abstract}
Background/Aims: Systemic lupus erythematosus (SLE) is a heterogeneous chronic inflammatory autoimmune disorder, in the pathogenesis of which miRNAs play a versatile function. The purpose of this study was to investigate the effect of miRNA-410 on the pathogenesis of SLE in T cells of SLE patients. Methods: Real-time PCR was used to test the mRNA levels of miRNA-410 in SLE patients and healthy controls. ELISA analysis was performed to examine the production levels of IL-10. Luciferase Assay was used to confirm the targeting effect of miRNA-410 on 3'UTR of STAT3 mRNA. Results: We found that the expression level of miR-410 in T cells of SLE patients was decreased comparing to that in healthy controls, whereas overexpression of miR-410 significantly reduced the expression levels of IL-10. Furthermore, miR-410 suppresses the transcription activity of STAT3 by binding directly to the 3 'UTR of STAT3 mRNA. Moreover, silence of STAT3 down regulated IL-10 expression in CD3+ $T$ cells. Conclusion: Our results demonstrate that miR-410 is the key regulatory factor in the pathogenesis of SLE by regulating the expression of IL-10 through targeting STAT3. These data suggest a novel function of miR-410 and bring new insight into understanding the complex mechanisms involved in SLE.

(C) 2016 The Author(s)

Published by S. Karger AG, Basel
\end{abstract}

\section{Introduction}

Systemic lupus erythematosus (SLE) is a heterogeneous chronic inflammatory autoimmune disorder, which is characterized by progressive involvement of multiple-organ systems with alternating clinical exacerbations and remissions $[1,2]$. The pathogenesis of SLE is complex and confusing, involving interactions between environmental and various 


\section{Cellular Physiology Cell Physiol Biochem 2016;39:303-315 \\ \begin{tabular}{l|l} 
and Biochemistry 10.1159/000445625 & $\begin{array}{l}\text { DO } 2016 \text { The Author(s). Published by S. Karger AG, Basel } \\
\text { www.karger.com/cpb }\end{array}$
\end{tabular} \\ Liu et al.: MiR-410 Down-Regulates Interleukin-10 by Targeting STAT3 in CD3+ T Cells}

of SLE

aspects of the immune system. Serum interleukin-10 (IL-10) levels have been found higher in SLE patients, and both spontaneous and mitogen-induced IL-10 production is increased in peripheral blood mononuclear cells (PBMCs) of SLE patients [3]. Moreover, it has been demonstrated that IL-10 levels are strongly implicated in the pathogenesis of SLE $[4,5]$. Except for IL-10, macrophages [6], hyper-activation of B and T cells, immune complex deposition with subsequent infiltration of neutrophils, reduced ability of immune complexes as well as apoptotic cell clearance, and defects in multiple immune regulatory networks, are found to contribute to organ inflammation and subsequent damage in SLE [7]. Also, Meroni et al. reported that new loci and genes were associated with the pathogenesis of SLE [8]. Current studies have clarified the aberrant molecular biological levels which were induced by environmental and epigenetic factors are thought to contribute to the pathogenesis of SLE [9]. Exploitation of these abnormalities has been proposed for development of therapeutic targets and biomarkers. Although several factors have been found to contribute to the pathogenesis of SLE, the mechanism of action and the target gene in SLE is still not fully understood. Thus, it makes sense to investigate the potential mechanism and screen the SLErelated gene in order to improve the diagnosis and the therapy of SLE.

MicroRNAs (miRNAs) are a class of 21- to 25-nucleotide single-stranded, endogenous, non-coding RNAs which regulate gene expression through binding to the mRNA $3^{\prime}$ untranslated. It has been implicated in diverse cellular processes including inflammation factors [10], auto-antibodies [11], cell immunity [12], cell apoptosis, proliferation as well as differentiation [13]. Also, miRNAs have been associated with other different areas such as the immune response [14], neural development, DNA repair, and oxidative stress response [15]. Notably, it is confirmed that lots of diseases including SLE have been found to be regulated by the expression level of miRNAs [16]. Recently, exponentially growing evidence demonstrates that miRNAs play a versatile function in the pathogenesis of SLE [17-20]. Previously, our team has searched for miRNAs that may be involved in the pathogenesis of SLE in PBMCs from SLE patients by microarray analysis [21]. It was found that a total of 29 miRNAs were down-regulated in PBMCs of SLE patients as compared with healthy controls, in which miRNA-410 was likely involved in the signaling transduction pathways in regulating the pathogenesis of SLE.

This study aimed at investigating the role of miRNA-410 in SLE T cells in the possible dysregulated biological processes and pathogenesis of SLE. We found that miRNA-410 by down-regulating the expression of IL-10 through targeting STAT3. This study brings new insight into clarifying the complex mechanisms involved in the pathogenesis of SLE and a novel biomarker for SLE diagnostics.

\section{Materials and Methods}

\section{Human subjects: SLE patients and healthy controls}

This research protocol was approved by Institutional Review Board of China Medical University, which all participants provided consent written informed. Twenty Chinese patients diagnosed with SLE were admitted to Shengjing Hospital of China Medical University and enrolled in our present study. They had never been treated with disease-modifying antirheumatic drugs or other immunosuppressive drugs. The diagnosis of the twenty patients was made according to the classification criteria of SLE developed by the American College of Rheumatology (Hochberg, 1997). Twenty healthy subjects who matched with the SLE patients by gender and age were recruited as controls.

PBLs (peripheral blood lymphocytes) and CD3+/CD4+ T cell isolation

PBLs were obtained from the SLE patients and healthy controls, using ethylenediaminetetraacetic acid (EDTA) as the anticoagulant. Phosphate buffered saline (PBS) was added into the samples at the radio of 1:1 before centrifugation. T cells were isolated by Ficoll-Hypaque Solution (Sigma-Aldrich, St. Louis, MO, USA) density gradient centrifugation from the venous blood. After cytometry, T cells $\left(10^{7}\right.$ cells $/ 80 \mu \mathrm{l}$ buffer) were resuspended in CD3+/CD4+ cells sorting magnetic beads buffer. CD3+ T cells were isolated by 


\section{Cellular Physiology Cell Physiol Biochem 2016;39:303-315 \begin{tabular}{l|l} 
DOI: 10.1159/000445625 & Ond Biochemistry \\
Published online: June 29, 2016 & $\begin{array}{l}\text { 2016 The Author(s). Published by S. Karger AG, Basel } \\
\text { www.karger.com/cpb }\end{array}$
\end{tabular} \\ Liu et al.: MiR-410 Down-Regulates Interleukin-10 by Targeting STAT3 in CD3+ T Cells of SLE}

magnetic-activated cell sorting (MACS) kit (CD3 MicroBeads, Miltenyi Biotec, Germany) according to the manufacturer's instructions. CD4+ T cells were also isolated by MACS kit (CD4 MicroBeads, Miltenyi Biotec, Germany). The $\mathrm{CD}^{+}$and CD4+ T cells collected were all cultured in Dulbecco's Modified Eagle's Medium (DMEM, Gibco ${ }^{\circledR}$, Grand Island, NY, USA) containing 10\% (v/v) heat-inactivated fetal bovine serum (FBS, HyClone Co., Logan, UT, USA).

\section{Vector construction}

Template cDNAs from our lab were used to construct vectors the oligonucleotide primers of which are listed as follows: mir-410 CDS: 5'-CCA GAA GCT TAT GTC CCA TCC GTC CTC A-3' (forward) and 5'-ACT TGG ATC CGC ACA ACC CTA GCA TCT TC-3' (reverse); The 3' untranslated region (3'UTR) of IL-10 was obtained by the amplification of genomic DNA using a forward primer (5'-CTC GCT AGC ACT TCT CTG GAG CAG ATA TT-3') and a reverse primer (5'-GGC GTC GAC TTA CGG TTC CTA TAT AAC G-3'). For the mutant construct, the seed sequence was mutated as follows: 5'-TGA CGA GGC TAA CAC AGA TGG-3' (forward) and 5'-GTG TTA GCC TCG TCA TTA ATA A-3' (reverse). The PCR product was cloned into the pUM-T simple vector (BioTeke Co., Beijing, China) and verifid by sequencing. All PCR reactions were performed using Life Express RTPCR machine (BIOER, Hangzhou, China). The entry clone plasmids, pcDNA3.1-mir-410 and pmirGLO-3'UTR were extracted using No endotoxin plasmid preparation kit (DP2802, BioTeke, Beijing, China) by inserting a T-mir-410/pcDNA3.1+ and a T-3'UTR, pmirGLO fragments, respectively.

\section{RNA interference}

To generate miR-410 overexpression and STAT3 silence CD3+ T cells, the clonal plasmids were harvested. For the anti-miR-410 group, a miR-410 inhibitor and a control siRNA were purched from Gene Pharma (Shanghai, China) and then transfected into CD3+ T cells. The diluted miRNA-410(-) plasmid, miRNA-410, siRNA(-), siRNA(STAT3), anti-miR-410(-) and anti-miR-410 plasmids were added into Lipofectamine $^{\circledR} 2000$ Reagent (1:1 ratio, Invitrogen, Grand Island, NY, USA) and incubated for $20 \mathrm{~min}$ at room temperature. After that, the complex was added to CD3+ T cells and incubated for $4 \mathrm{~h}$ at $37^{\circ} \mathrm{C}$. Cells were continue to be planted in 6-wells plates with Dulbecco's Modified Eagle's Medium (DMEM, Gibco ${ }^{\circledR}$, Grand Island, NY, USA) containing 10\% (v/v) heat-inactivated fetal bovine serum (FBS, HyClone Co., Logan, UT, USA) for $24 \mathrm{~h}$ at $37^{\circ} \mathrm{C}$. Later, 50ng/ml PMA (P8139, Sigma-Aldrich, St. Louis, MO, USA) and $1 \mu \mathrm{M}$ ionomycin (1-0634, Sigma-Aldrich, St. Louis, MO, USA) were added into the cells for stimulation. Cells were collected after $24 \mathrm{~h}$ incubation.

\section{Real-time PCR}

The expression levels of miR-410 and IL-10 in different groups were validated using Real-time (RT) PCR in all cell samples (twenty SLE patients and twenty healthy controls). Total RNA was extracted using High purity total RNA extraction kit (RP1201, BioTeke Co, Beijing, China) according to the manufacturer's protocol. cDNAs were synthesized from total RNAs using Super M-MLV reverse transcriptase kit (PR6502, BioTeke, Beijing, China). The Reverse Transcription products were then amplified for PCR. Primers used in RT-PCR were as follows: miR-410: CCG CAC GAT ATA ACA CAG ATG (sense) and GTG CAG GGT CCG AGG TAT TC (antisense); U6: CTC GCT TCG GCA GCA CA (sense) and AAC GCT TCA CGA ATT TGC GT (antisense); IL-10: CGA TTT AGA AAG AAG CCC AA (sense) and TCA ACA GCT AGA AAG CGT GGT (antisense); STAT3: AAG GAC ATC AGC GGT AAG AC (sense) and GAG ATA GAC CAG TGG AGA CAC (antisense); $\beta$-actin: CTT AGT TGC GTT ACA CCC TTT CTT G (sense) and CTG TCA CCT TCA CCG TTC CAG TTT (antisense). SYBR Green (Solarbio, Beijing, China) was used for RT-PCR. Forward primer, reverse primer, and $10 \mathrm{ng}$ template cDNA was prepared; the reaction condition for the RT was as follows: $25^{\circ} \mathrm{C}$ for $10 \mathrm{~min}, 42^{\circ} \mathrm{C}$ for $50 \mathrm{~min}$, and $95^{\circ} \mathrm{C}$ $5 \mathrm{~min}$. The PCR amplification conditions were: $95^{\circ} \mathrm{C}$ for $10 \mathrm{~min}, 40$ cycles of $95^{\circ} \mathrm{C}$ for $10 \mathrm{~s}, 60^{\circ} \mathrm{C}$ for $20 \mathrm{~s}$ and $72^{\circ} \mathrm{C}$ for $30 \mathrm{~s}$, then $4^{\circ} \mathrm{C}$ for $5 \mathrm{~min}$. The PCR results were verified by varying the number of PCR cycles for each cDNA and set of primers. PCR reaction was performed using Exicycler ${ }^{\mathrm{TM}} 96$ RT-PCR machine (Bioneer, Daejeon, Korea) with $\beta$-actin as a control. RT-PCR was performed at least in quadruplicate.

\section{Western blot analysis}

Anti-IL-10, anti-STAT3, anti-p-STAT3 and anti- $\beta$-actin were obtained from Santa cruz Biotechnology, Inc. (Dallas, Texas, USA). For western blot analyses, protein content was determined using BCA Protein Assay Kit (Beyotime Co., Shanghai, China) following manufacturer's instructions. $40 \mu \mathrm{g}$ of proteins were separated 


\section{Cellular Physiology Cell Physiol Biochem 2016;39:303-315

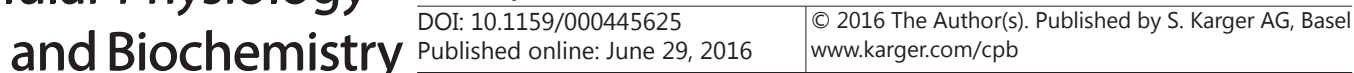 \\ Liu et al.: MiR-410 Down-Regulates Interleukin-10 by Targeting STAT3 in CD3+ T Cells \\ of SLE}

using 9\% sodium dodecyl sulfate-polyacrylamide gel electrophoresis (SDS-PAGE) and transferred to polyvinylidene difluoride (PVDF) membranes (Millipore Co., Billerica, MA, USA). The filters were hybridized with the appropriate primary antibodies overnight at $4^{\circ} \mathrm{C}$. The relative amounts of the transferred proteins were quantified by scanning the autoradiographic films with a gel densitometer and normalized to the corresponding $\beta$-actin level. Quantitative analysis for Western blot was made by Gel-Pro-Analyzer software.

ELISA

The concentration of IL-10 in CD3+ T cells was determined with the Interleukin-10 (IL-10) ELISA Assay Kit (WHB-Bio., Shanghai, China) according to the manufacturer's protocol. All the samples and the standards diluted into different concentrations were added into the wells at the volume of $50 \mu \mathrm{l}$. The absorbance of the wells was measured using microplate reader (BIOTEK, Winooski, VT, USA) at $450 \mathrm{~nm}$, and the concentration levels of IL-10 was calculated from the standard curve.

\section{Luciferase Assay}

Luciferase assays were measured using Dual-Luciferase ${ }^{\circledR}$ Reporter (DLR ${ }^{\mathrm{m}+\mathrm{T}}$ ) Assay System (E1910, Promega, Madison, WI, USA) according to the manufacturer's instructions. In brief, the cells were cultured in 6-well plates and transfected with vectors, miR-410 plasmids or the miRNA mutant control using Lipofectamine 2000 reagent (Invitrogen, Grand Island, NY, USA). The fiefl and Renilla luciferase activities were measured at $24 \mathrm{~h}$ after transfection using the dual-luciferase assay system (Promega, Madison, WI, USA).

\section{Statistical analysis}

Data were presented as the mean values \pm standard deviation (SD) from at least three experiments. Statistical comparisons were analyzed by Bonferroni's multiple comparison tests or analysis of variance. $\mathrm{P}<0.05$ was considered as a statistically significant difference.

\section{Results}

Dysregulated miR-410 expression in CD3+ T cells of SLE patients comparing to Healthy controls

PBLs from 20 Chinese SLE patients and 20 Healthy controls were collected for this study. CD3+ T cells cells were isolated by magnetic-activated cell sorting (MACS) kit. RT-PCR analysis was used to explore miRNA-410 expression level in CD3+ T cells from SLE patients and Healthy controls. We found that the expression level of miRNA-410 in SLE patients was lower than that in Healthy controls $\left({ }^{* *} \mathrm{P}<0.01\right.$, Fig. 1A). As we have known, in the subsets of $\mathrm{T}$ cells, CD4+ T cells which are important immune cells in the immune system, have been also used to study the microRNA regulation in SLE $[22,23]$. Also, the miRNA-410 level was tested in CD4+ T cells (Fig. 1B). As we excepted, the expression level of miRNA-410 in CD4 + T cells of SLE patients was lower than that in Healthy controls $\left({ }^{* *} \mathrm{P}<0.01\right)$. It was also found that the miR-410 level was negatively correlated with the percentage of CD4+ T cells in SLE patients (Fig. 1C\&D). Together with the results in Fig. 1A, our observations indicated that miR-410 expression is decreased in T cells especially in CD4+ T cells of SLE patients comparing with Healthy controls suggesting the close relations between miRNA-410 expression in $\mathrm{T}$ cells and the pathogenesis of SLE. For investigating the effects of miR-410 in overall T cells functions, CD3+ T cells were used to investigate the role of miR-410 in SLE for further study.

MiR-410 down-regulated the expression of IL-10 in CD3+ T cells of SLE patients

Cytokines are reported to play an important and diverse role in the pathogenesis of SLE, in which the level of IL-10 is a key factor for disease activity [24]. To evaluate whether the level of IL-10 in CD3+ T cells from SLE patients is associated with miR-410, first, miR410 overexperssion vectors as well as negative control vectors were transfected into CD3+ $\mathrm{T}$ cells. The mRNA levels of IL-10 in CD3+ T, miR-NC and miR-410 cells from SLE patients and healthy controls were examined by RT-PCR analysis. Data of healthy control groups in Fig. 2A showed that, the mRNA level of IL-10 was decreased slightly without statistical 


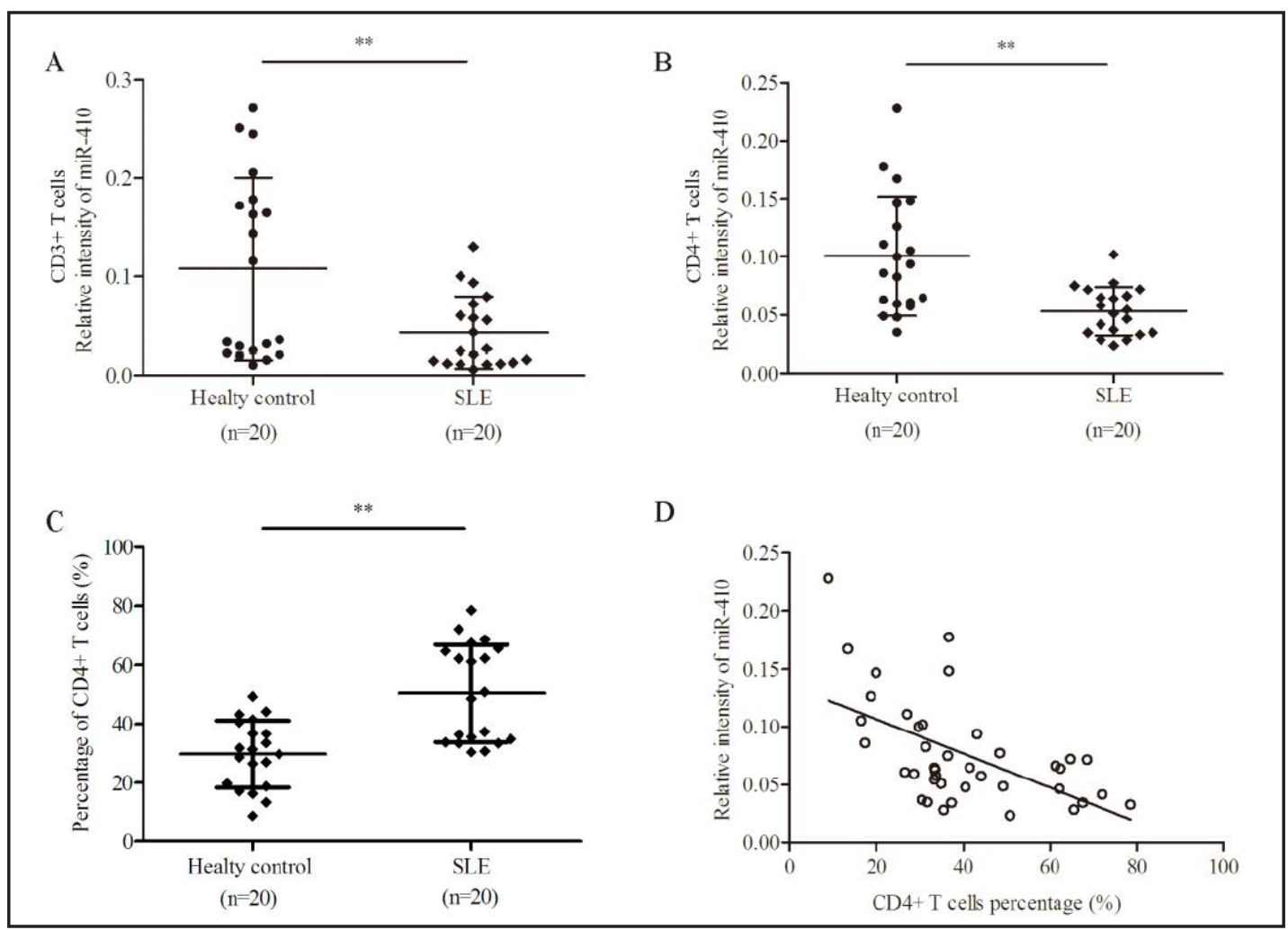

Fig. 1. The expression levels of miR-410 in T cells of SLE patients and healthy control. (A) Relative miR-410 mRNA levels in CD3+ T cells of SLE patients and healthy control were tested by RT-PCR. (B) The expression levels of miR-410 in CD4+ T cells of SLE patients compared with healthy control. (C) The percentage change in CD4+ T cells in SLE patients compared with that in healthy control by FACS analysis. (D) Correlation between expression level of miR-410 and percentage of CD4+ T cells in SLE patients. The miR-410 level is negatively correlated with the percentage CD4+ T cells. $r=0.5648$. SLE, systemic lupus erythematosus. ${ }^{* *} \mathrm{P}<0.01$ versus Healthy control group.

differences in miR-410 CD3+ T cells comparing with that in control group; However, the mRNA of IL-10 was significantly increased in CD3+ T cells of SLE groups (from $1.00 \pm 0.00$ in Healty control to $3.69 \pm 0.39$ in SLE group, \&\& $P<0.01$ ), while overexpression of miR410 down-regulated the mRNA level of IL-10 in half. As seen in T cells of SLE groups, there were no obvious differences between SLE and miR-NC cells, the relative mRNA level of IL10 between miR-NC and miR-410 group was decreased from $3.76 \pm 0.44$ to $1.85 \pm 0.29{ }^{{ }^{* *}} \mathrm{P}$ $<0.01$ ). The same trends with the result of RT-PCR, Western blot analysis showed that the protein level of IL-10 expression in SLE group was twice higher than that in Healthy control (from $1.00 \pm 0.00$ in control group to $2.16 \pm 0.26$ in SLE group, ${ }^{\& \&} \mathrm{P}<0.01$ ). Overexpression of miR-410 significantly down-regulated the protein level of IL-10 in miR-410 cells. The quantitative value of gray intensity analysis of IL-10 between miR-NC and miR-410 was 2.14 \pm 0.26 vs. $0.72 \pm 0.13\left({ }^{* *} \mathrm{P}<0.01\right.$, Fig. $\left.2 \mathrm{~B}\right)$. In addition, the IL- 10 levels in supernatant of CD3+ $\mathrm{T}$ cells between Healty control groups and SLE groups were tested using ELISA analysis, and data showed that the IL-10 level in supernatant of SLE group was increased more than three times than that in Healthy control (from $35.94 \pm 4.14$ to $140.61 \pm 16.95$, \& $P<0.01$ ). Likewise, overexpression of miR-410 decreased the IL-10 level in supernatant (from 144.94 \pm 11.29 in miR-NC group to $55.72 \pm 6.74$ in miR-410 group, ${ }^{* *} \mathrm{P}<0.01$, Fig. $2 \mathrm{C}$ ). These data suggest that miRNA-410 decreased the expression levels of IL-10 in CD3+ T cells.

To figure out the role of miRNA-410 in the decrease of IL-10, anti-miRNA-410 (CD3+ T cells transfected with miRNA-410 inhibitor vector), miR-NC (CD3+ T cells transfected with an empty vector) and CD3+ T cells were used to compare the expression levels of IL-10 in

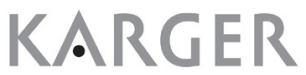


A
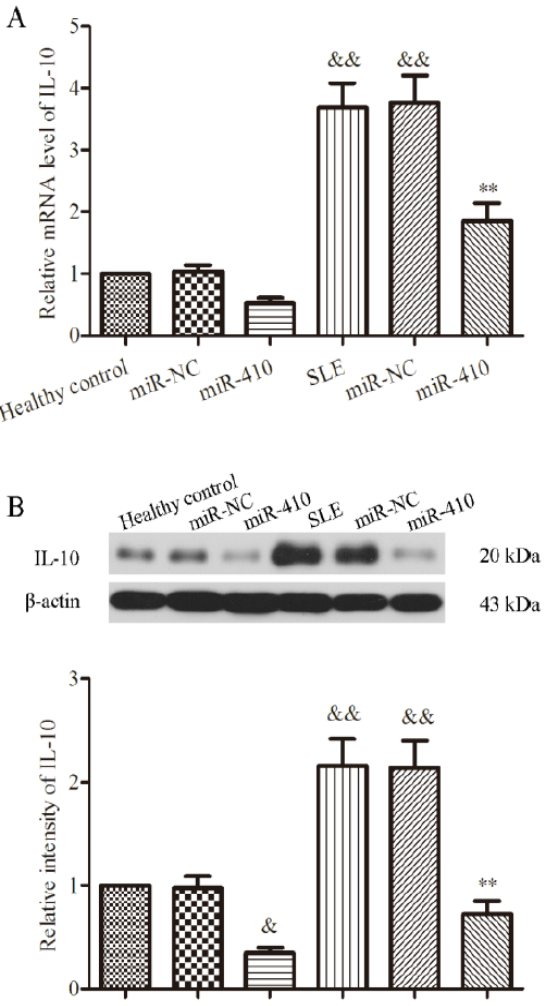

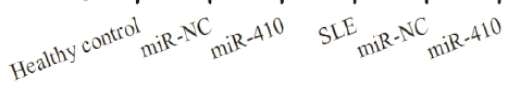

$\mathrm{C}$

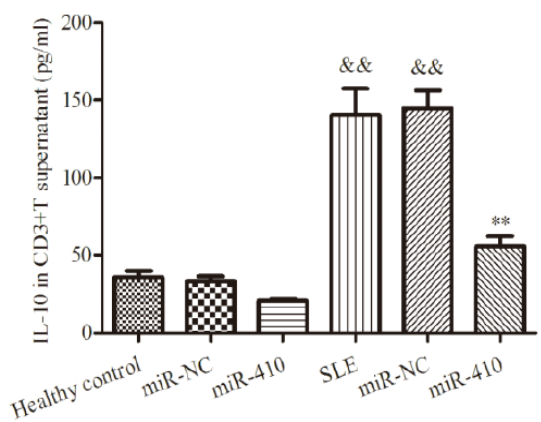

D

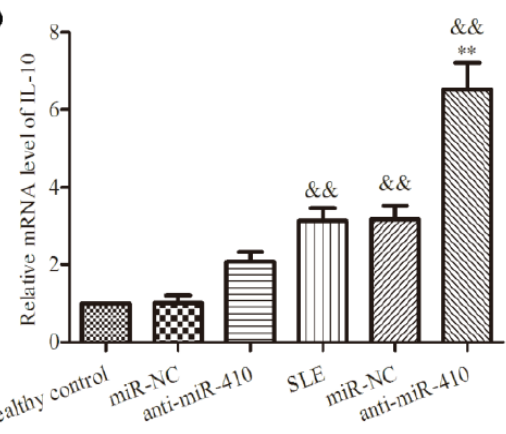

E

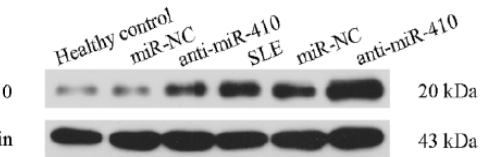

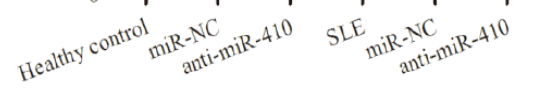

$\mathrm{F}$

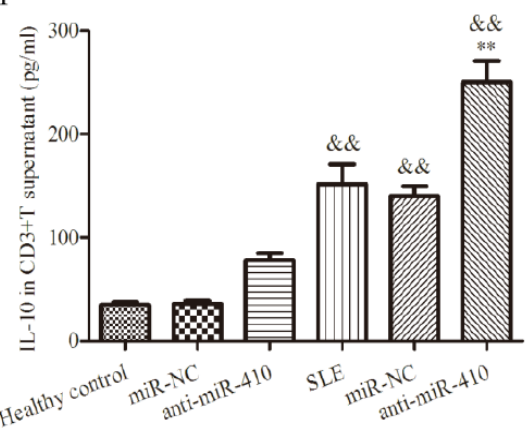

Fig. 2. miR-410 regulated the expression of IL-10 in CD3+ T cells of SLE patients. Overexpression of miR410 down-regulated the expression levels of IL-10 in CD3+ T cells. CD3+ T Cells from both Healthy controls and SLE patients were transfected with miR-410/empty vectors and then stimulated with $50 \mathrm{ng} / \mathrm{ml}$ PMA as well as $1 \mu \mathrm{M}$ ionomycin for $24 \mathrm{~h}$. Relative mRNA levels of IL-10 were tested by RT-PCR analysis (A). The protein levels of IL-10 were tested by Western blot analysis (B, above) and the quantitative analysis of gray intensity was calculated and showed in (B, below). The concentration of IL-10 in CD3+ T supernatant was tested by ELISA analysis (C). Inhibition of miR-410 increased the expression levels of IL-10 in CD3+ T cells. CD3+ T Cells from both Healthy controls and SLE patients were transfected with miR-410 inhibitor or empty vectors. Relative mRNA levels of IL-10 were tested by RT-PCR analysis (D). The protein levels of IL-10 were tested by Western blot analysis (E, above) and the quantitative analysis of gray intensity was calculated and showed in (E, below). The concentration of IL-10 in CD3+ T supernatant was tested by ELISA analysis $(\mathrm{F}) .{ }^{\&} \mathrm{P}<0.05$ or ${ }^{\& \&} \mathrm{P}<0.01$ versus Healthy control group; ${ }^{*} \mathrm{P}<0.05$ or ${ }^{* *} \mathrm{P}<0.01$ versus SLE miR-NC group. miR-NC, CD3+ T cells from Healthy control/SLE patients transfected with an empty (negative control) vector; miR-410, CD3+ T cells from Healthy control/SLE patients transfected with a miR-410 vector; anti-miR-410, CD3+ T cells from Healthy control/SLE patients transfected with a miR-410 inhibitor vector. 


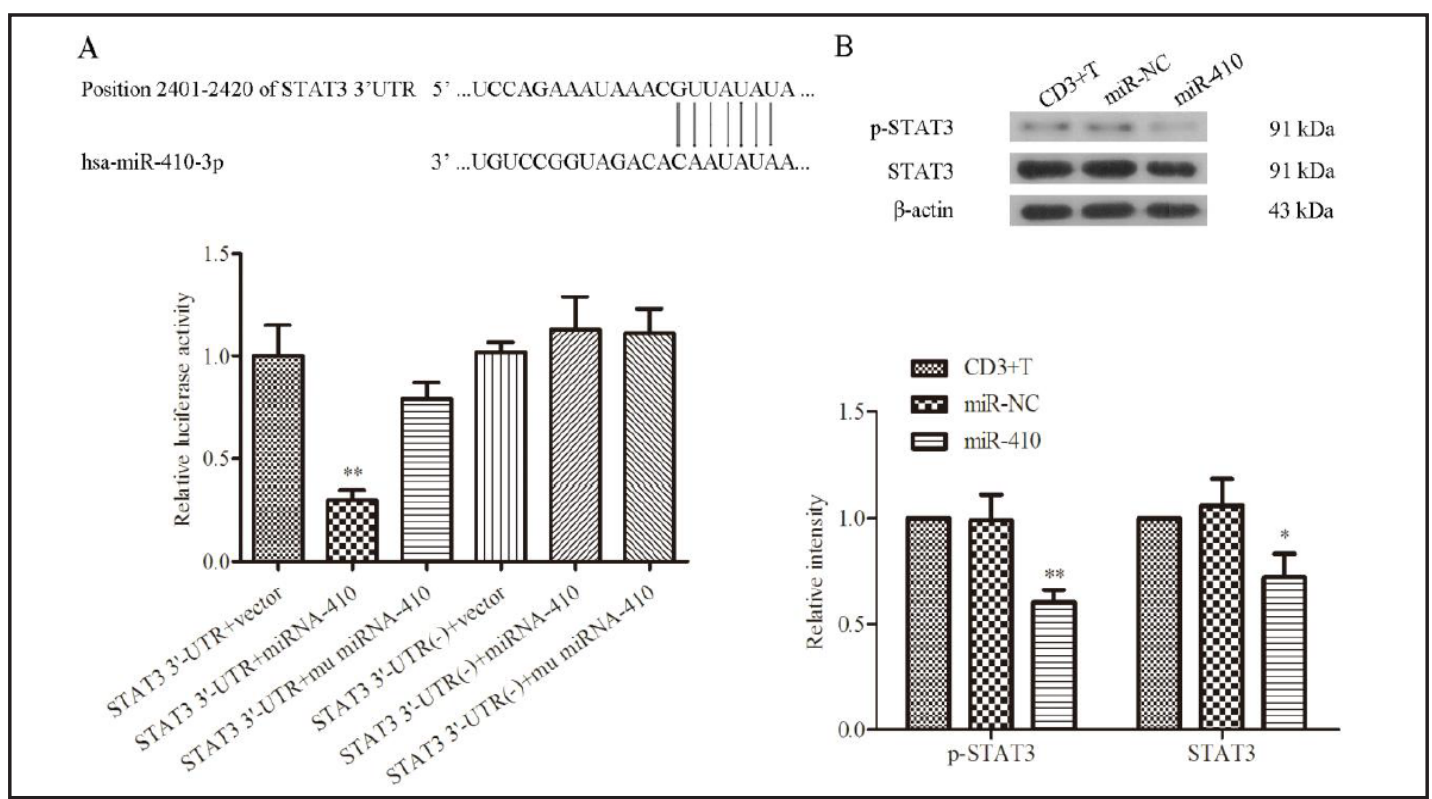

Fig. 3. miR-410 is a key mediator in the regulation of STAT3 expression in CD3+ T cells of SLE patients. (A) Transfection with miR-410 dramatically reduced the luciferase activity in CD3+ T cells transfected with the luciferase reporter vector containing the 3'-UTR of STAT3 compared with those transfected with miRNA control groups. STAT 3'-UTR, cells transfected with the vector containing the 3'-UTR of STAT3; vector, cells transfected with an ampty vector; miR-410, cells transfected with a miR-410 vector; mu miR-410, cells transfected with a mutant miR-410 vector. (B) Overexpression of miR-410 down-regulated the phosphorylation and the expression level of STAT3 by Western blot anylysis and the quantitative analysis of gray intensity was calculated and showed in (B).

both Healthy control groups and SLE groups. As seen in the results of Healthy control groups, there were no obvious differences between control and anti-miR-410 group, while in the SLE groups, the mRNA level of IL-10 was obviously increased in anti-miR-410 CD3+ T cells of SLE patients (from $3.17 \pm 0.36$ in miR-NC cells to $6.53 \pm 0.67$ in anti-miR-410 cells, ${ }^{* *} \mathrm{P}<0.01$, \&\& $\mathrm{P}<0.01$, Fig. 2D). Western blot analysis showed that the expression levels of IL-10 in antimiRNA-410 cells of SLE patients was also increased from $2.24 \pm 0.30$ to $4.41 \pm 0.58\left({ }^{* *} \mathrm{P}<\right.$ 0.01 , \& $\mathrm{P}<0.01$, Fig. 2E). Meanwhile, the IL-10 level in supernatant of anti-miRNA-410 cells of SLE patients was twice higher than that in miR-NC cells $(140.14 \pm 9.18$ in miR-NC cells vs. $250.02 \pm 20.56$ in anti-miRNA-410 cells, ${ }^{* *} \mathrm{P}<0.01$, \&\& $\mathrm{P}<0.01$, Fig. $\left.2 \mathrm{~F}\right)$. These data suggest that miRNA-410 regulated the expression levels of IL-10 in CD3+ T cells.

MiR-410 is a key mediator in the regulation of STAT3 expression in CD3+ T cells of SLE patients

It is reported that STAT3 is involved in SLE and the target of miR-124. Over-expression of miR-155 significantly increased IL-21 mediated STAT signaling in SLE [22, 25]. In order to find whether STAT3 is the target of miR-410, luciferase reporter assay was performed. The intact 3' UTR of STAT3 was cloned into luciferase reporter plasmids, which were used for co-transfection with miR-410 or mutant-miR-410 as control into CD3+ T cells. Figure 3A showed that miR-410 significantly suppressed the luciferase activity of the 3' UTR reporter for STAT3, whereas mutations of the miR-410 binding sites abrogated the suppression of luciferase expression (Fig. 3A), which suggests that miR-410 suppresses the transcription activity of STAT3 by directly bind to the 3 'UTR of STAT3 mRNA.

Furthermore, the phosphorylation and expression levels of STAT3 in the CD3+ T, miR-NC (CD3+ T cells transfected with an empty vector) and miR-410 (CD3+ T cells transfected with a miR-410 vector) cells were determined by Western blot analysis (Fig. 3B). As we expected, 


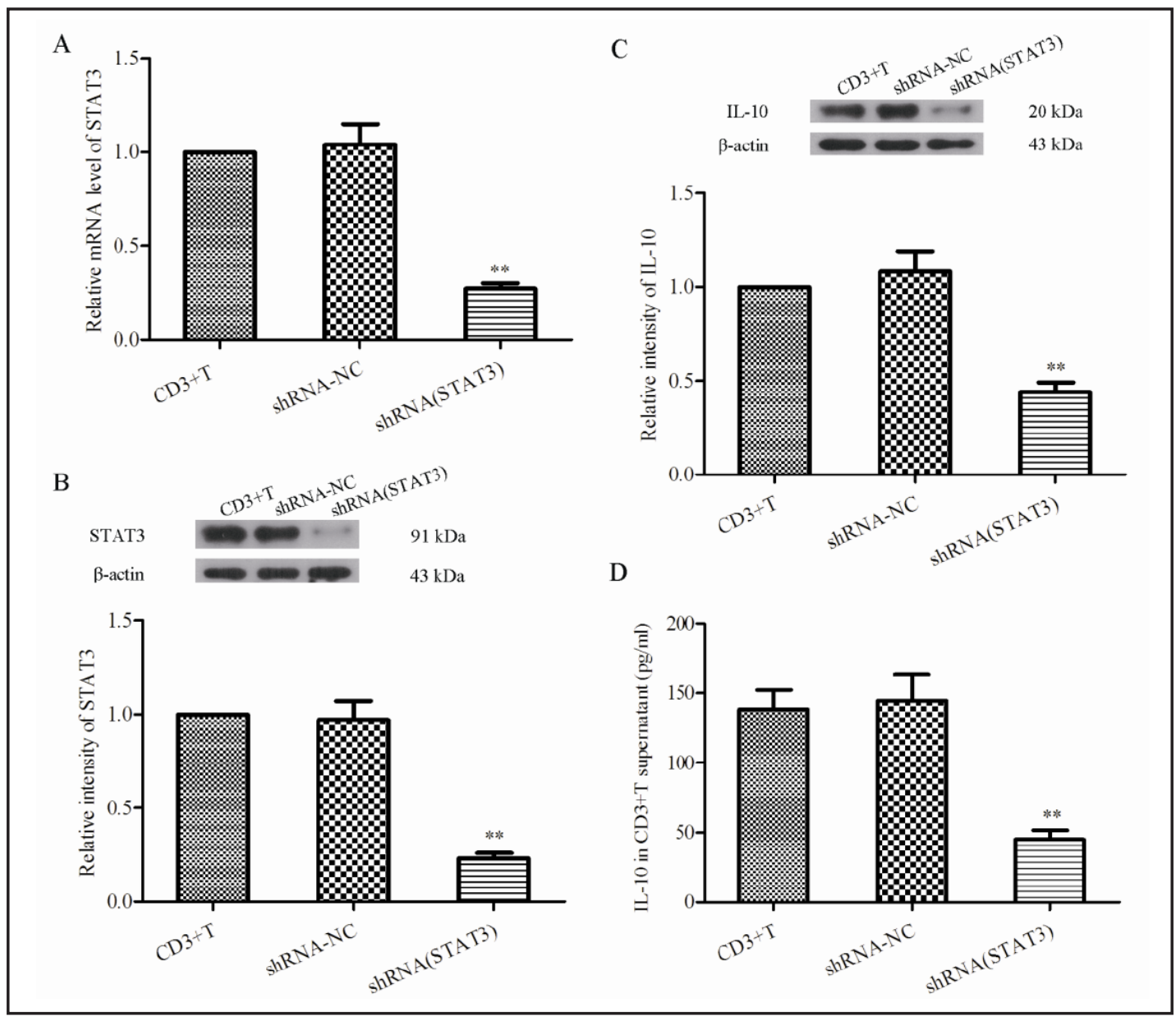

Fig. 4. Silence of STAT3 down-regulated the expression of IL-10 in CD3+ T cells. Cells were first transfected with shRNA(STAT3)/shRNA-NC vectors and then stimulated with $50 \mathrm{ng} / \mathrm{ml}$ PMA as well as $1 \mu \mathrm{M}$ ionomycin for 24 h. (A) The relative expression levels of STAT3 mRNA in CD3+ T, shRNA-NC, shRNA(STAT3) cells were tested by RT-PCR. (B) The expression levels of STAT3 protein. The expression levels of STAT3 protein in CD3+ T, shRNA-NC, shRNA(STAT3) cells were compared by Western blot analysis and the quantitative analysis of gray intensity was determined. (C) Silence of STAT3 down-regulated the expression of IL-10. The protein levels of IL-10 were tested by Western blot analysis (C, above) and the quantitative analysis of gray intensity was calculated and showed in (C, below). The concentrations of IL-10 in supernatant of CD3+ T, shRNA-NC, shRNA(STAT3) cells were tested by ELISA analysis (D) ${ }^{* *} \mathrm{P}<0.01$ versus shRNA-NC group. CD3+ T, CD3+ T cells of SLE patients without RNA interference; shRNA-NC, CD3+ T cells transfected with an empty vector; shRNA(STAT3), CD3+ T cells transfected with STAT3-silence vector.

both the phosphorylation and the expression levels of STAT3 in miR-410 cells was lower than that in miR-NC or CD3+ T cells. There were no obvious differences between CD3+ T and miR-NC cells, the quantitative value of gray intensity analysis of p-STAT3 and STAT3 between miR-NC and CD3+ T was $0.99 \pm 0.12$ vs. $0.60 \pm 0.06$ and $1.06 \pm 0.12$ vs. $0.72 \pm 0.11\left({ }^{* *} \mathrm{P}<0.01\right.$, Fig. 3B). Taken together, these results indicate that miR-410 regulated the expression levels of STAT3 by directly bind to the 3 'UTR of STAT3 mRNA in CD3+ T cells of SLE.

Silence of STAT3 down-regulated the expression of IL-10 in CD3+ T cells

It has been confirmed that T cell-derived IL-10 mediates myeloid inflammation in part via macrophage STAT3 in the inflammatory bowel diseases (IBDs) [26]. To investigate the relationship between STAT3 and IL-10 in CD3+ T cells of SLE, STAT3 was silenced in 


\section{Cellular Physiology Cell Physiol Biochem 2016;39:303-315

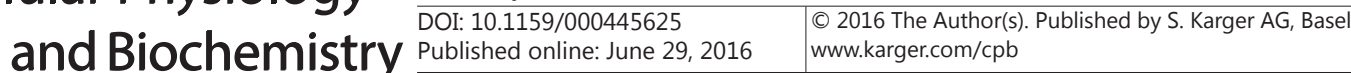 \\ Liu et al.: MiR-410 Down-Regulates Interleukin-10 by Targeting STAT3 in CD3+ T Cells}

of SLE

CD3+ T cells by RNA interference. The mRNA level of STAT3 in CD3+ T, shRNA-NC (CD3+ T cells transfected with an empty vector) and shRNA(STAT3) (CD3+ T cells transfected with STAT3 silence vectors) cells were tested using RT-PCR anylysis. As shown in Fig. 4A, the mRNA expression levels of STAT3 were 4 -fold lower in the shRNA(STAT3) cells $(0.27 \pm 0.03)$, as compared with the CD3+ T $(1.0 \pm 0.00)$ and shRNA-NC $(1.04 \pm 0.11)$ cells. In addition, the expression levels of STAT3 in CD3+ T, shRNA-NC and shRNA(STAT3) cells were also examined by Western blot analysis. Fig. 4B showed that there was little STAT3 expression in the shRNA(STAT3) cells. The quantitative analysis of gray intensity revealed that STAT3 expression levels were also 4 -fold lower in shRNA(STAT3) $(0.23 \pm 0.03)$ cells, as compared with the CD3+ T $(1.00 \pm 0.00)$ and shRNA-NC $(0.97 \pm 0.10)$ cells (Fig. 4B, $\mathrm{P}<0.01)$.

Western blot analysis was used to test the expression level of IL-10 after silence of STAT3 in CD3+ T cells. As we expected, silence of STAT3 suppressed the production of IL10 in shRNA(STAT3) cells. There were no obvious differences between CD3+ T and shRNANC cells, the quantitative value of gray intensity analysis of IL-10 between shRNA-NC and shRNA(STAT3) was $1.08 \pm 0.11$ vs. $0.44 \pm 0.05\left({ }^{* *} \mathrm{P}<0.01\right.$, Fig. $\left.4 \mathrm{C}\right)$. Identical to the result of Western blot analysis, silence of STAT3 also decreased the IL-10 level in supernatant of shRNA(STAT3) cells comparing to that in CD3+ T cells by ELISA analysis, and data showed that the IL-10 level in supernatant between shRNA-NC and shRNA(STAT3) was $144.64 \pm$ 19.15 vs. $45.12 \pm 6.43\left({ }^{* *} \mathrm{P}<0.01\right.$, Fig. 4D). These data suggest that the production level of IL-10 was mediated by STAT3 in CD3+ T cells.

\section{Discussion}

SLE is considered as a potentially lethal systemic autoimmune disease with multi-organ damage. Since autoantibodies are elevated in the pathogenesis of SLE, auto-reactive B-cells as well as T-cells have been recognized to be important of it, in which $\mathrm{T}$ cells are critical players in SLE pathophysiology as they regulate B cell responses and also infiltrate target tissues, leading to tissue damage [27]. Therefore, they have been studied most intensively and extensively $[28,29]$. On the other hand, recent discoveries have indicated that the aberrant expression of miRNAs is associated with the pathogenesis of SLE [30], which gives us new insights into the pathogenesis of SLE and might lead to the finding of new therapeutic targets.

In this study we investigated the expression of miR-410 in CD3+ T cells of twenty Chinese SLE patients and healthy controls to elucidate the role of miR-410 in the pathogenesis of SLE. We found that levels of miR-410 in CD3+ T cells of SLE patients were decreased comparing to that in healthy controls (Fig. 1). Moreover, the production level of IL-10 was increased in CD3+ T cells of SLE patients whereas overexpression of miR-410 decreased the IL-10 level in CD3+ T cells (Fig. 2). Also, we found that miR-410 regulated the levels of STAT3 directly in CD3+ T cells of SLE patients (Fig. 3). Luciferase activity analysis showed that miR-410 was found to directly bind to the 3 'UTR of STAT3 (Fig. 3) while silence of STAT3 decreased the level of IL-10 by Western blot analysis in CD3+ T cells of SLE patients (Fig. 4). Our results indicated that miR-410 plays an important role in the pathogenesis and development of SLE.

Exponentially growing evidence demonstrates that miRNAs play a versatile function in multiple biological and metabolic progresses although the functions are still unclear for a majority of identified miRNAs [31-34]. Recent investigations demonstrate that dysregulated expression of microRNA has been reported to be associated with the pathogenesis of SLE [35]. We found that the mRNA expression levels of miRNA-410 in SLE patients were lower than that in healthy controls, which showed that miRNA-410 may be a key factor in SLE development. Although, in previous studies, it was known that 29 miRNAs were identified differentially expressed in SLE patients, and the expression of miR-1246 was decreased in the activated B cells from SLE patients through the AKT-P53 signaling pathway [36], miR410 was the first time to show its involvement in the deregulated signaling pathways related to SLE patients [21]. The miRNA also has been reported to function as a tumor suppressor 


\section{Cellular Physiology Cell Physiol Biochem 2016;39:303-315 \\ and Biochemistry Published online: June 29, $2016 \quad \begin{aligned} & \text { DOI: 10.1159/000445625 } 2016 \text { The Author(s). Published by S. Karger AG, Basel } \\ & \text { www.karger.com/cpb }\end{aligned}$ \\ Liu et al.: MiR-410 Down-Regulates Interleukin-10 by Targeting STAT3 in CD3+ T Cells}

of SLE

in human gliomas [37]. Thus, we can speculate that the expression of miR- 410 is essential to the pathogenesis of SLE patients. However, it is needed to figure out the precise function of miR-410 and to unravel its regulatory mechanisms in SLE.

IL-10 is found to be an immune-regulatory cytokine with pro- and anti-inflammatory functions, which plays a vital role in the pathogenesis of autoimmune disorders such as SLE, including regulation of the differentiation and the growth of B cells and auto-antibody production [38]. Although there were no changes in serum IL-10 and IL-17 levels in B10 cell transferred MRL/lpr SLE mice [39], in various cells, including T lymphocytes and monocytes/ macrophages, IL-10 reduces the expression of pro-inflammatory cytokines, thus suppressing effector phenotypes [40]. However, the effects of IL-10 on B cells appear contradictory. IL-10 is reported to promote B-cell survival, differentiation, proliferation and antibody production in inflammation and disease [41]. It was found that increased level of IL-10 expression in SLE patients correlates with disease activity and antibody production, while blockade of IL-10 corrected the dysregulated cytokine responses [41] and facilitated clinical improvement in SLE patients [42]. Recently, this contradiction has been at least partially resolved. Increasing studies reported that serum IL-10 levels have been found higher in SLE patients and reduced expression of the IL-10 in SLE T cells could alter the effects of IL-10 on activated or memory $\mathrm{T}$ cells which are major sources of pro-inflammatory cytokines [23]. In the present study, we found that both the mRNA and the expression level of IL-10 were reduced by overexpression of miR-410 in CD3+ T cells of SLE patients. Also, the serum IL-10 level in CD3+ T cells was decreased in miR-410 overexpressing group. Furthermore, inhibition of miR-410 by transfection with miRNA-410 inhibitor reversed both the serum and the cellular levels of IL10 in CD3+ T cells. These results confirmed that miRNA-410 regulated the expression levels of IL-10 in CD3+ T cells of SLE patients.

We then studied the mechanism of how miRNA-410 regulated the expression of IL-10 in SLE. STAT3 is a critical member of the STAT transcription factor family. It is reported that overexpression of miR-155 in CD4+ T cells increased STAT3 phosphorylation in response to IL-21 treatment in SLE [22]. Also, Hedrich et al. reported that in lupus T cells, the increasing level of IL-10 was promoted by STAT3 [43]. However, how these events are mechanistically regulated in SLE and involvement, if any, of miRNAs in these events is poorly understood. In our study, we found that miR-410 regulated the expression levels of STAT3 by directly bind to the 3 'UTR of STAT3 mRNA in CD3+ T cells of SLE. Moreover, overexpression of miRNA-410 decreased the phosphorylation as well as the expression level of STAT3 in CD3+ T cells of SLE patients. In SLE, the phosphorylation of STAT3 forms a homodimer, which translocates to the nucleus where it initiates transcription of target genes including the IL-10, IL-17A and IL-21. This forms an autocrine loop for their production via STAT3 phosphorylation [22, 43]. Thus, we can conclude that miRNA-410 regulated the inactivation of STAT3 by suppressing the phosphorylation as well as the expression level of STAT3 via directly targeting STAT3 gene in CD3+ T cells of SLE patients.

In T cells of SLE patients, activation of STAT3 is increased, resulting in enhanced recruitment to regulatory regions and competitive replacement of STAT5, subsequently promoting IL-10 expression [43]. STAT3 was reported to play a central role in the regulation of pro-inflammatory effector cytokines, including IL-17A and IL-10, promoting effector T-cell phenotypes [44]. Furthermore, STAT3 is involved in IL-10 induction, which resulted in the proposal of STAT3-blocking strategies in autoimmune disorders and cancer. None of those studies, however, targeted the effect of STAT3 on the expression of IL-10 in SLE. In our study, we found that silence of STAT3 promoted the decrease of IL-10 both in CD3+ T cells and in cellular supernatant in of SLE patients. These results showed that STAT3 was the key factor in regulating the expression of IL-10 in CD3+ T cells. It was reported that the STAT signaling pathways are regulated through multiple levels, the intronic STAT-responsive element was reported to regulated the production of IL-10 in murine natural killer ells (through STAT4) and human T cells (through STAT5) [45, 46]. And the STAT-induced STAT inhibitors are confirmed to form a key negative feedback loop for regulation and attenuation of STAT signals. Cheng et al. also reported that the expression of STAT3 in esophageal cancer 


\section{Cellular Physiology Cell Physiol Biochem 2016;39:303-315 \begin{tabular}{c|c|c|} 
DOI: 10.1159/000445625 & $\begin{array}{l}\text { O 2016 The Author(s). Published by S. Karger AG, Basel } \\
\text { www.karger.com/cpb }\end{array}$
\end{tabular} \\ Liu et al.: MiR-410 Down-Regulates Interleukin-10 by Targeting STAT3 in CD3+ T Cells of SLE}

cell lines was regulated by miRNA-21 through, at least partially, targeting STAT3 signaling pathway [25]. The particular mechanism of how the STAT3 signaling pathway is involved in the SLE is expected to be clarified by further research. Thus, our study confirmed that miRNA-410 regulated the expression of IL-10 via directly targeting STAT3 in CD3+ T cells of SLE patients.

\section{Conclusion}

In conclusion, we demonstrate for the first time that miR-410 is the key regulatory factor in the pathogenesis of SLE, which regulated the expression of IL-10 via directly targeting STAT3 in CD3+ T cells of SLE patients. These data suggest a novel function and a therapeutic application of miR-410 in SLE.

\section{Acknowledgements}

This study was supported by grants from the National Natural Science Foundation of China (No. 81501408) and the Doctoral Startup Foundation of Liaoning Province (No. 201501019).

\section{Disclosure Statement}

The authors declare that there are no conflicts of interest.

\section{References}

1 Talaat RM, Mohamed SF, Bassyouni IH, Raouf AA: Th1/Th2/Th17/Treg cytokine imbalance in systemic lupus erythematosus (SLE) patients: Correlation with disease activity. Cytokine 2015;72:146-153.

2 Liang B, Gardner DB, Griswold DE, Bugelski PJ, Song XY: Anti-interleukin-6 monoclonal antibody inhibits autoimmune responses in a murine model of systemic lupus erythematosus. Immunology 2006;119:296305.

3 Llorente L, Richaud-Patin Y, Fior R, Alcocer-Varela J, Wijdenes J, Fourrier B M, Galanaud P, Emilie D: In vivo production of interleukin-10 by non-T cells in rheumatoid arthritis, Sjogren's syndrome, and systemic lupus erythematosus. A potential mechanism of B lymphocyte hyperactivity and autoimmunity. Arthritis Rheum 1994;37:1647-1655.

4 Park YB, Lee SK, Kim DS, Lee J, Lee CH, Song CH: Elevated interleukin-10 levels correlated with disease activity in systemic lupus erythematosus. Clin Exp Rheumatol 1998;16:283-288.

5 Houssiau FA, Lefebvre C, Vanden Berghe M, Lambert M, Devogelaer JP, Renauld JC: Serum interleukin 10 titers in systemic lupus erythematosus reflect disease activity. Lupus 1995;4:393-395.

6 Li F, Yang Y, Zhu X, Huang L, Xu J: Macrophage Polarization Modulates Development of Systemic Lupus Erythematosus. Cell Physiol Biochem 2015;37:1279-1288.

7 Crispin JC, Liossis SN, Kis-Toth K, Lieberman LA, Kyttaris VC, Juang YT, Tsokos GC: Pathogenesis of human systemic lupus erythematosus: recent advances. Trends Mol Med 2010;16:47-57.

8 Meroni PL, Shoenfeld Y: Systemic lupus erythematosus and the SLE galaxy. Autoimmun Rev 2010;10:1-2.

9 Rahman A, Isenberg DA: Systemic lupus erythematosus. N Engl J Med 2008;358:929-939.

10 Jiang X, Xue M, Fu Z, Ji C, Guo X, Zhu L, Xu L, Pang L, Xu M, Qu H: Insight into the effects of adipose tissue inflammation factors on miR-378 expression and the underlying mechanism. Cell Physiol Biochem 2014;33:1778-1788. 


\section{Cellular Physiology Cell Physiol Biochem 2016;39:303-315 \begin{tabular}{ll|l} 
and Biochemistry $\begin{array}{l}\text { DOI: 10.1159/000445625 } \\
\text { Published online: June 29, } 2016\end{array}$ & $\begin{array}{l}\text { C } 2016 \text { The Author(s). Published by S. Karger AG, Basel } \\
\text { www.karger.com/cpb }\end{array}$
\end{tabular} \\ Liu et al.: MiR-410 Down-Regulates Interleukin-10 by Targeting STAT3 in CD3 + T Cells of SLE}

11 Ivanova VV, Khaiboullina SF, Cherenkova EE, Martynova EV, Nevzorova TA, Kunst MA, Sibgatullin TB, Maksudova AN, Oliveira PJ, Lombardi VC, Palotas A, Rizvanov AA: Differential immuno-reactivity to genomic DNA, RNA and mitochondrial DNA is associated with auto-immunity. Cell Physiol Biochem 2014;34:2200-2208.

12 Yang S, Li F, Jia S, Zhang K, Jiang W, Shang Y, Chang K, Deng S, Chen M: Early secreted antigen ESAT-6 of Mycobacterium Tuberculosis promotes apoptosis of macrophages via targeting the microRNA155-SOCS1 interaction. Cell Physiol Biochem 2015;35:1276-1288.

13 Allegra A, Alonci A, Campo S, Penna G, Petrungaro A, Gerace D, Musolino C: Circulating microRNAs: new biomarkers in diagnosis, prognosis and treatment of cancer (review). Int J Oncol 2012;41:1897-1912.

14 Dou L, Wang S, Sui X, Meng X, Shen T, Huang X, Guo J, Fang W, Man Y, Xi J, Li J: MiR-301a mediates the effect of IL-6 on the AKT/GSK pathway and hepatic glycogenesis by regulating PTEN expression. Cell Physiol Biochem 2015;35:1413-1424.

15 Zhang B, Farwell MA: microRNAs: a new emerging class of players for disease diagnostics and gene therapy. J Cell Mol Med 2008;12:3-21.

16 Fiorucci G, Chiantore MV, Mangino G, Percario ZA, Affabris E, Romeo G: Cancer regulator microRNA: potential relevance in diagnosis, prognosis and treatment of cancer. Curr Med Chem 2012;19:461-474.

17 Wu H, Zhao M, Chang C, Lu Q: The Real Culprit in Systemic Lupus Erythematosus: Abnormal Epigenetic Regulation. Int J Mol Sci 2015;16:11013-11033.

18 Qu B, Shen N: miRNAs in the Pathogenesis of Systemic Lupus Erythematosus. Int J Mol Sci 2015;16:95579572.

19 Kaga H, Komatsuda A, Omokawa A, Ito M, Teshima K, Tagawa H, Sawada K, Wakui H: Down-regulated expression of miR-155, miR-17, and miR-181b, and up-regulated expression of activation-induced cytidine deaminase and interferon-alpha in PBMCs from patients with SLE. Mod Rheumatol 2015;1-25.

20 Deng X, Su Y, Wu H, Wu R, Zhang P, Dai Y, Chan TM, Zhao M, Lu Q: The role of microRNAs in autoimmune diseases with skin involvement. Scand J Immunol 2015;81:153-165.

21 Liu D, Zhao H, Zhao S, Wang X: MicroRNA expression profiles of peripheral blood mononuclear cells in patients with systemic lupus erythematosus. Acta Histochem 2014;116:891-897.

22 Rasmussen TK, Andersen T, Bak RO, Yiu G, Sorensen CM, Stengaard-Pedersen K, Mikkelsen J G, Utz P J, Holm CK, Deleuran B: Overexpression of microRNA-155 increases IL-21 mediated STAT3 signaling and IL21 production in systemic lupus erythematosus. Arthritis Res Ther 2015;17:154.

23 Cui HD, Qi ZM, Yang LL, Qi L, Zhang N, Zhang XL, Du SY, Jiang Y: Interleukin-10 receptor expression and signalling were down-regulated in $\mathrm{CD} 4(+) \mathrm{T}$ cells of lupus nephritis patients. Clin Exp Immunol 2011;165:163-171.

24 Doria A, Ghirardello A, Iaccarino L, Zampieri S, Punzi L, Tarricone E, Ruffatti A, Sulli A, Sarzi-Puttini PC, Gambari PF, Cutolo M: Pregnancy, cytokines, and disease activity in systemic lupus erythematosus. Arthritis Rheum 2004;51:989-995.

25 Cheng Y, Li Y, Nian Y, Liu D, Dai F, Zhang J: STAT3 is involved in miR-124-mediated suppressive effects on esophageal cancer cells. BMC Cancer 2015;15:306.

26 Kamanaka M, Kim S T, Wan YY, Sutterwala FS, Lara-Tejero M, Galan J E, Harhaj E, Flavell RA: Expression of interleukin-10 in intestinal lymphocytes detected by an interleukin-10 reporter knockin tiger mouse. Immunity 2006;25:941-952.

27 Moulton VR, Tsokos GC: Abnormalities of T cell signaling in systemic lupus erythematosus. Arthritis Res Ther 2011;13:207.

28 Coca A, Sanz I: Updates on B-cell immunotherapies for systemic lupus erythematosus and Sjogren's syndrome. Curr Opin Rheumatol 2012;24:451-456.

29 Crispin JC, Kyttaris VC, Terhorst C, Tsokos GC: T cells as therapeutic targets in SLE. Nat Rev Rheumatol 2010;6:317-325.

30 Shen N, Liang D, Tang Y, de Vries N, Tak PP: MicroRNAs--novel regulators of systemic lupus erythematosus pathogenesis. Nat Rev Rheumatol 2012;8:701-709.

31 Ambros V, Chen X: The regulation of genes and genomes by small RNAs. Development 2007;134:16351641.

32 Zhang B, Pan X, Cobb GP, Anderson TA: Plant microRNA: a small regulatory molecule with big impact. Dev Biol 2006;289:3-16. 


\section{Cellular Physiology Cell Physiol Biochem 2016;39:303-315 \begin{tabular}{ll|l} 
DOI: 10.1159/000445625 & $\begin{array}{l}\text { O 2016 The Author(s). Published by S. Karger AG, Basel } \\
\text { www.karger.com/cpb }\end{array}$ \\
\cline { 2 - 3 }
\end{tabular} \\ Liu et al.: MiR-410 Down-Regulates Interleukin-10 by Targeting STAT3 in CD3+ T Cells of SLE}

33 Zhang B, Pan X, Anderson TA: MicroRNA: a new player in stem cells. J Cell Physiol 2006;209:266-269.

34 Bentwich I, Avniel A, Karov Y, Aharonov R, Gilad S, Barad O, Barzilai A, Einat P, Einav U, Meiri E, Sharon E, Spector Y, Bentwich Z: Identification of hundreds of conserved and nonconserved human microRNAs. Nat Genet 2005;37:766-770.

35 Tang Q, Yang Y, Zhao M, Liang G, Wu H, Liu Q, Xie Y, Li D, Dai Y, Yung S, Chan TM, Lu Q: Mycophenolic acid upregulates miR-142-3P/5P and miR-146a in lupus CD4+T cells. Lupus 2015;24:935-942.

36 Luo S, Liu Y, Liang G, Zhao M, Wu H, Liang Y, Qiu X, Tan Y, Dai Y, Yung S, Chan TM, Lu Q: The role of microRNA-1246 in the regulation of B cell activation and the pathogenesis of systemic lupus erythematosus. Clin Epigenetics 2015;7:24.

37 Chen L, Zhang J, Feng Y, Li R, Sun X, Du W, Piao X, Wang H, Yang D, Sun Y, Li X, Jiang T, Kang C, Li Y, Jiang C: MiR-410 regulates MET to influence the proliferation and invasion of glioma. Int J Biochem Cell Biol 2012;44:1711-1717.

38 Rousset F, Garcia E, Defrance T, Peronne C, Vezzio N, Hsu DH, Kastelein R, Moore KW, Banchereau J: Interleukin 10 is a potent growth and differentiation factor for activated human B lymphocytes. Proc Natl Acad Sci USA 1992;89:1890-1893.

39 Yu M, Song Y, Zhu M X, Liang W, Long Q Ding P W, Xie Y, Liao YH, Yuan J: B10 Cells Ameliorate the Progression of Lupus Nephritis by Attenuating Glomerular Endothelial Cell Injury. Cell Physiol Biochem 2015;36:2161-2169.

40 Hofmann SR, Rosen-Wolff A, Tsokos GC, Hedrich CM: Biological properties and regulation of IL-10 related cytokines and their contribution to autoimmune disease and tissue injury. Clin Immunol 2012;143:116127.

41 Hedrich CM, Bream JH: Cell type-specific regulation of IL-10 expression in inflammation and disease. Immunol Res 2010;47:185-206.

42 Llorente L, Richaud-Patin Y, Garcia-Padilla C, Claret E, Jakez-Ocampo J, Cardiel M H, Alcocer-Varela J, Grangeot-Keros L, Alarcon-Segovia D, Wijdenes J, Galanaud P, Emilie D: Clinical and biologic effects of antiinterleukin-10 monoclonal antibody administration in systemic lupus erythematosus. Arthritis Rheum 2000;43:1790-1800.

43 Hedrich CM, Rauen T, Apostolidis SA, Grammatikos AP, Rodriguez Rodriguez N, Ioannidis C, Kyttaris VC, Crispin JC, Tsokos GC: Stat3 promotes IL-10 expression in lupus T cells through trans-activation and chromatin remodeling. Proc Natl Acad Sci USA 2014;111:13457-13462.

44 Chen Z, O'Shea JJ: Th17 cells: a new fate for differentiating helper T cells. Immunol Res 2008;41:87-102.

45 Tsuji-Takayama K, Suzuki M, Yamamoto M, Harashima A, Okochi A, Otani T, Inoue T, Sugimoto A, Toraya T, Takeuchi M, Yamasaki F, Nakamura S, Kibata M: The production of IL-10 by human regulatory T cells is enhanced by IL-2 through a STAT5-responsive intronic enhancer in the IL-10 locus. J Immunol 2008;181:3897-3905.

46 Grant LR, Yao ZJ, Hedrich CM, Wang F, Moorthy A, Wilson K, Ranatunga D, Bream JH: Stat4-dependent, T-bet-independent regulation of IL-10 in NK cells. Genes Immun 2008;9:316-327. 